\title{
AVALIAÇÃO DA TECNOLOGIA DO ULTRASSOM PORTÁTIL E SUA CORRELAÇÃO COM O PERCENTUAL DE GORDURA OBTIDO PELAS DOBRAS CUTÂNEAS EM ADULTOS JOVENS
}

\author{
EVALUATION OF PORTABLE ULTRASOUND TECHNOLOGY AND THE CORRELATION WITH THE \\ FAT PERCENTAGE THROUGH SKINFOLDS IN YOUNG ADULTS
}

\author{
Eddy Krueger ${ }^{\mathrm{a}^{*}}$, Leandra Ulbricht ${ }^{\mathrm{b}^{*}}$, Wagner Ripka ${ }^{\mathrm{c}^{*}}$, Eduardo Borba Neves ${ }^{\mathrm{d}^{*}}$

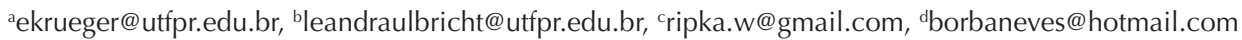 \\ *Universidade Tecnológica Federal do Paraná - Curitiba (PR), Brasil
}

Data de recebimento do artigo: 15/01/2014

Data de aceite do artigo: 03/06/2015

\section{RESUMO}

Introduçáo: O objetivo deste estudo foi comparar e correlacionar os resultados da estimativa do percentual de gordura e das dobras cutâneas por ultrassom (US) portátil e por adipômetro científico em adultos jovens. Métodos: No ano de 2012, foram avaliados 120 militares do sexo masculino, divididos de acordo com o valor do índice de massa corporal (IMC). Foram coletados: peso, estatura, dobras cutâneas (DC) e US em nove pontos (tríceps braquial, subescapular, bíceps braquial, peitoral, axilar média, abdominal, suprailíaca, coxa anterior e panturilha). A análise estatística foi realizada com os testes não paramétricos de correlação de Spearman $(\rho)$ e um teste entre dois grupos pareados no Wilcoxon Signed Ranks Test. Resultados: Para a correlação dos percentuais de gordura obtidos por meio de sete dobras cutâneas e dos valores obtidos pela medição com o US, foram obtidas os seguintes resultados: $\rho=0,421$ (IMC< $25 \mathrm{~kg} / \mathrm{m}^{2}, \mathrm{~N}=94$ ); $\rho=0,419$ (IMC $\geq 25 \mathrm{~kg} / \mathrm{m}^{2}, \mathrm{~N}=26$ ); $\rho=0,514$ (IMC geral, $\mathrm{N}=120$ ), Todas as correlaçôes apresentaram uma estatística significante $(\mathrm{p}<0,05)$. Os pontos com maior correlação foram o da coxa $(\rho=0,65)$ e do tríceps $(\rho=0,63)$. Conclusáo: Ao compararmos os percentuais de gordura estimados pelo ultrassom portátil e pelo adipômetro, verificaramos correlaçóes significativas entre todos os pontos avaliados. Contudo, os valores de US foram inferiores aos obtidos por DC em todos os pontos analisados, exceto o bíceps braquial. Em vista disso, recomendamos que sejam realizados estudos que comparem o US portátil com os raios $\mathrm{x}$ de dupla energia, para podermos efetivamente verificar se existe uma correlaçáo entre o diagnóstico obtido a partir dessa nova tecnologia com uma técnica considerada padrão-ouro.

Palavras-chave: Composição corporal; dobras cutâneas; ultrassonografia.

\section{ABSTRACT}

Introduction: The aim of this study was to compare and correlate the results of fat percentage by skin fold thickness using portable ultrasound (US) and scientific adipometer in adults. Methods: During 2012 we evaluated 120 military men, divided according to the value of the body mass index (BMI). We collected: weight, height, SF and US in nine points (triceps, subscapular, biceps, chest, medium axillary, abdominal, supra iliac, anterior thigh and calf). Statistical analysis was performed using nonparametric tests as Spearman correlation coefficient and paired with Wilcoxon Signed Ranks Test. Results: For the correlation of the percentage of fat obtained through seven skinfolds and values obtained by measuring it with US, the following results were obtained: $\rho=0.421\left(\mathrm{BMI}<25 \mathrm{~kg} / \mathrm{m}^{2}, \mathrm{~N}=94\right) ; \rho=0.419$ $\left(\mathrm{BMI} \geq 25 \mathrm{~kg} / \mathrm{m}^{2}, \mathrm{~N}=26\right) ; \rho=0.514$ (general BMI, $\mathrm{N}=120$ ), where all correlations were statistically significant $(p<0.05)$. Points with the highest correlations were the thigh $(\rho=0.65)$ and triceps $(\rho=0.63)$. Conclusion: Comparing the percentage of fat estimated between portable ultrasound and adipometer, there were significant correlations between all points assessed, but with lower US values than skin fold thickness at every point analyzed (except for the biceps brachialis). We recommend that future studies should be carried out comparing portable US with Dual-energy X-ray absorptiometry, so we could ef- 
fectively verify if there is a correlation between the diagnoses obtained from this new technology with a gold standard technique.

Keywords: Body composition; skinfold thickness; ultrasonography.

\section{Introdução}

O uso da técnica de ultrassonografia para a avaliação da gordura subcutânea é comum em animais há algumas décadas. Em seres humanos, as pesquisas iniciaram a partir da década de $1960^{1}$. Em um recente estudo publicado em 2013, Wagner ${ }^{2}$ apontava que a fidedignidade da técnica registrada pelo ultrassom (US) pode se igualar à conseguida por outros instrumentos de medidas de dobras cutâneas (DC). Dessa forma, o US necessita de pesquisas em diferentes grupos de participantes, para que seja testada a sua eficiência na prática clínica ${ }^{3}$.

Num estudo realizado em $1967^{4}$, os autores compararam a estimativa de composiçáo corporal por meio de US e de DC. Os autores observaram diferenças entre os dois métodos. A correlação encontrada entre DC e US foi de $\mathrm{r}=0,81 \pm 0,60$. Boothe et $\mathrm{al}^{4}{ }^{4}$ destacaram como principal aspecto do seu estudo a diferença entre os resultados obtidos entre essas duas mensuraçóes.

No início da década de 1980, foi conduzida uma pesquisa por Fanelli e Kuczmarski ${ }^{5}$, a qual envolveu 124 homens com idade entre 18 e 30 anos, e comparou a composição corporal obtida por DC, US $(7,0 \mathrm{MHz})$ e pesagem hidrostática $(\mathrm{PH})$. As mensuraçóes de gordura subcutânea com o US e as DC foram realizadas em sete pontos anatômicos: (1) tríceps; (2) subescapular; (3) bíceps; (4) abdominal; (5) suprailíaca; (6) coxa e (7) panturrilha. Os resultados mostraram uma boa correlação para todos os pontos, sendo as mais elevadas para tríceps $(\mathrm{r}=0,81)$, abdominal $(\mathrm{r}=0,86)$ e coxa $(\mathrm{r}=0,87)$. Os autores compararam os valores encontrados pelos US e pela $\mathrm{PH}$ em cada sítio anatômico, e verificaram altas correlaçóes nos dois métodos. As melhores correlações entre o US e a PH ocorreram nas dobras de abdômen e coxa.

O que justificaria o uso do US portátil para a avaliação do percentual de gordura seria portabilidade do equipamento e a facilidade de seu manuseio por iniciantes, pois o equipamento BX2000 (BodyMetrix IntelaMetrix Inc.) é facilmente conectado a um microcomputador, e calcula a espessura da camada de gordura e seu percentual corporal automaticamente.

Johnson et al. ${ }^{6}$ realizaram um estudo transversal, com a avaliação de 84 adultos com média de $23 \pm 4,7$ anos, para comparar os resultados do US portátil com a absorciometria de raios-X de dupla energia (DXA), no qual foi detectada uma discordância entre esses métodos na estimativa do percentual de gordura.
Nesse sentido, com a evolução da tecnologia atual, o presente trabalho teve por objetivo comparar e correlacionar os resultados da estimativa do percentual de gordura e das dobras cutâneas (DC) por ultrassom (US) portátil e por adipômetro científico em adultos jovens.

\section{Métodos}

Este estudo seguiu os aspectos éticos recomendados pela Resolução no 196/96 (atualizada pela Resolução no 466/2012) sobre pesquisas envolvendo seres humanos, bem como os princípios éticos contidos na Declaração de Helsinki (1964, reformulada em 1975, 1983, 1989, 1996 e 2000), e foi aprovado pelo Comitê de Ética em Pesquisa da Universidade Federal do Paraná sob o protocolo $\mathrm{n}^{\circ}$ 091/09. A amostra foi composta por $120 \mathrm{su}-$ jeitos do sexo masculino, recrutados e avaliados no ano de 2012. Os voluntários foram selecionados entre os militares pertencentes ao Exército Brasileiro, e sediados no município de Curitiba. Todos os participantes assinaram o Termo de Consentimento Livre e Esclarecido, autorizando a coleta e a utilização de seus dados.

Para a mensuração da massa corporal, os voluntários vestiam uma roupa leve (apenas bermuda) e permaneciam sobre a balança, imóveis. A estatura foi aferida com os voluntários descalços, em pé, com as pernas estendidas e juntas, os calcanhares apoiados no solo e a cabeça orientada para o plano de Frankfurt.

Para a avaliação antropométrica, foram utilizados os seguintes materiais: adipômetro científico (Cescorf) calibrado; balança digital (Wiso W801") com capacidade de 0-180 kg e com graduação de $100 \mathrm{~g}$; e um estadiômetro (WCS Woody Compact). A ultrassonografia foi feita por meio do aparelho BX2000 (BodyMetrix IntelaMetrix Inc.) acoplado em um microcomputador.

Para a coleta de dados, foram realizadas as marcaçóes dos nove sítios anatômicos, utilizados com uma caneta dermográfica: abdome (AB); axilar média (AM); bíceps braquial (BI); coxa anterior (CX); panturrilha pedial (PM); peitoral (PT); subescapular (SB); suprailíaco (SI) e tríceps braquial (TR). A posição anatômica dos pontos seguiu a metodologia proposta por Jackson e Pollock ${ }^{7}$, e o valor considerado foi a média de três avaliaçôes consecutivas nos dois métodos, DC e US. Para o cálculo do percentual de gordura, foram utilizadas as equaçôes de Jackson e Pollock, para a densidade corporal e de $\operatorname{Siri}^{8}$, para a estimativa do percentual de gordura. 
O equipamento BX2000 (BodyMetrix - IntelaMetrix ${ }^{\oplus}$ Inc.) opera com uma frequência de $2,5 \mathrm{MHz}$, e foi conectado a um notebook (Pentium dual-core, $2,0 \mathrm{MHz}$ ) que calculou automaticamente o percentual de gordura corporal após a coleta das dobras supracitadas.

Além da análise do total da amostra como um único grupo, realizamos uma estratificação dos dados avaliados pelo IMC, a fim de testar a aplicabilidade do US em sujeitos com classificação de IMC normal e com sobrepeso/obesidade.

$\mathrm{O}$ estudo utilizou a estatística descritiva, com medidas de posição e dispersão para a caracterização da amostra e da estatística inferencial, para a correlação das variáveis e a verificação de significância. Para a análise da correlação entre os valores em milímetros $(\mathrm{mm})$ obtidos pelo adipômetro e do equipamento de ultrassom (US), recorremos ao coeficiente de correlação não paramétrico de Spearman, após a realização do teste de Kolmogorov-Smirnov. O Wilcoxon Signed Ranks Test foi utilizado para verificar a diferença entre as medianas das medidas de DC e US.

\section{Resultados}

Foram avaliados 120 militares, todos do sexo masculino, com média de idade de 18,72 $\pm 0,59$ anos. As demais características antropométricas foram: massa corporal, 70,15 $\pm 9,37 \mathrm{~kg}$; estatura, $1,74 \pm 0,06$ metros; IMC, $23,05 \pm 2,72 \mathrm{~kg} / \mathrm{m}^{2}$ e percentual de gordura, $12,31 \pm 5,07 \%$. A Tabela 1 apresenta os valores desses indicadores dentro dos grupos IMC $<25$ e IMC $\geq 25$.

Os resultados contidos nas Figuras 1, 2 e 3 mostram os valores a partir da leitura do adipômetro com o valor apresentado pelo US. Recorremos a uma estratificação do IMC dos avaliados, a fim de testar a aplicabilidade do US nas populaçóes normal e com sobrepeso/obesidade.

Tabela 1: Caracterização antropométrica de 120 sujeitos, Curitiba, 2012.

\begin{tabular}{ccccccc}
\hline & & \multicolumn{2}{c}{ IMC $<25$} & \multicolumn{2}{c}{ IMC $\geq 25$} & \multicolumn{2}{c}{ Geral } \\
\cline { 2 - 7 } Variáveis & $\mathbf{N}$ & Média \pm DP & N & Média \pm DP & N & Média \pm DP \\
\hline Idade (ano) & 94 & $18,73 \pm 0,57$ & 26 & $18,69 \pm 0,67$ & 120 & $18,72 \pm 0,59$ \\
Massa (kg) & 94 & $66,94 \pm 6,81$ & 26 & $81,76 \pm 8,15$ & 120 & $70,15 \pm 9,37$ \\
Altura (m) & 94 & $1,74 \pm 0,05$ & 26 & $1,73 \pm 0,07$ & 120 & $1,74 \pm 0,06$ \\
IMC (kg/m $)$ & 94 & $21,95 \pm 1,80$ & 26 & $27,06 \pm 1,40$ & 120 & $23,05 \pm 2,72$ \\
Gordura (\%) & 94 & $10,84 \pm 3,62$ & 26 & $17,60 \pm 6,03$ & 120 & $12,31 \pm 5,07$ \\
\hline
\end{tabular}

IMC: índice de massa corporal, DP: desvio-padrão.

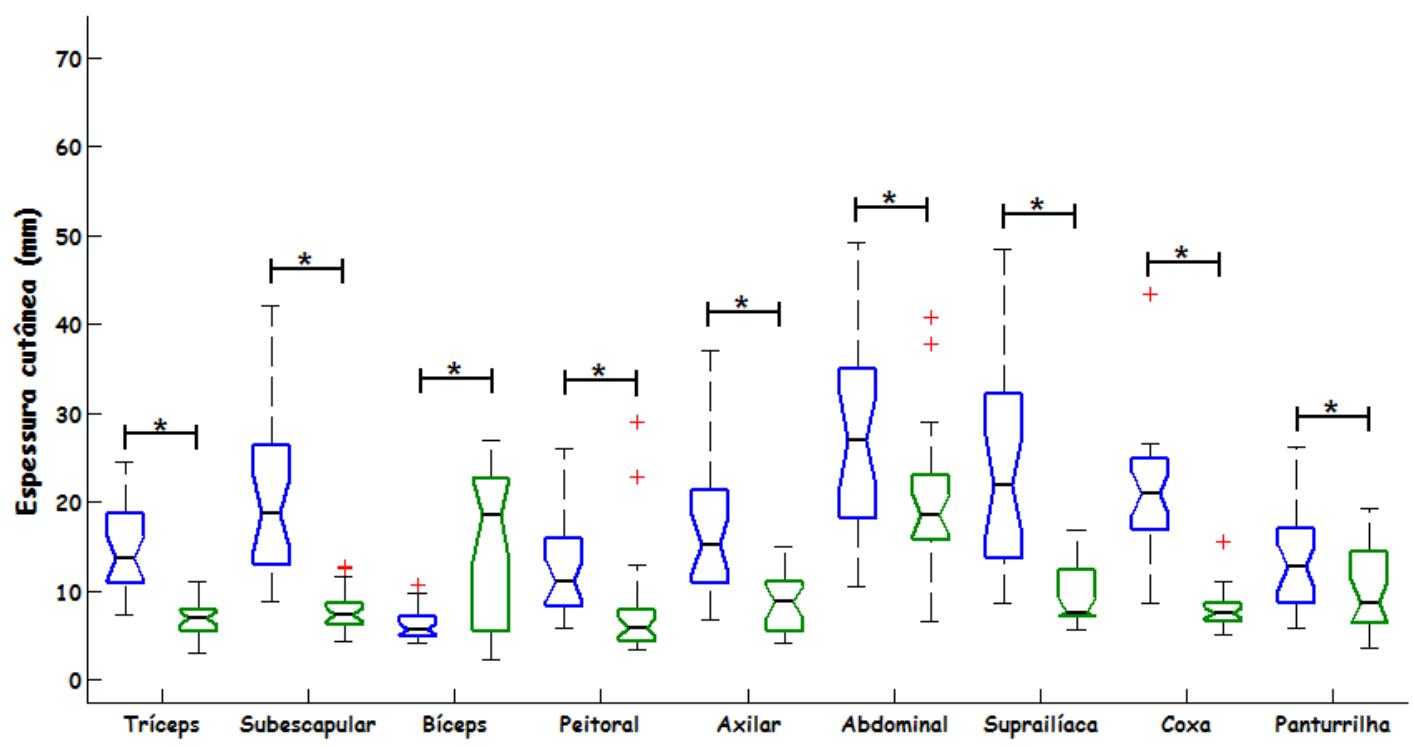

Figura 1: Gráfico do tipo boxplot da espessura cutânea registrada pelo adipômetro (cor azul) e pelo ultrassom (cor verde) em voluntários com IMC>25 kg/m² (N=26).+: outliers. *: $\mathrm{p} \leq 0,05$. 


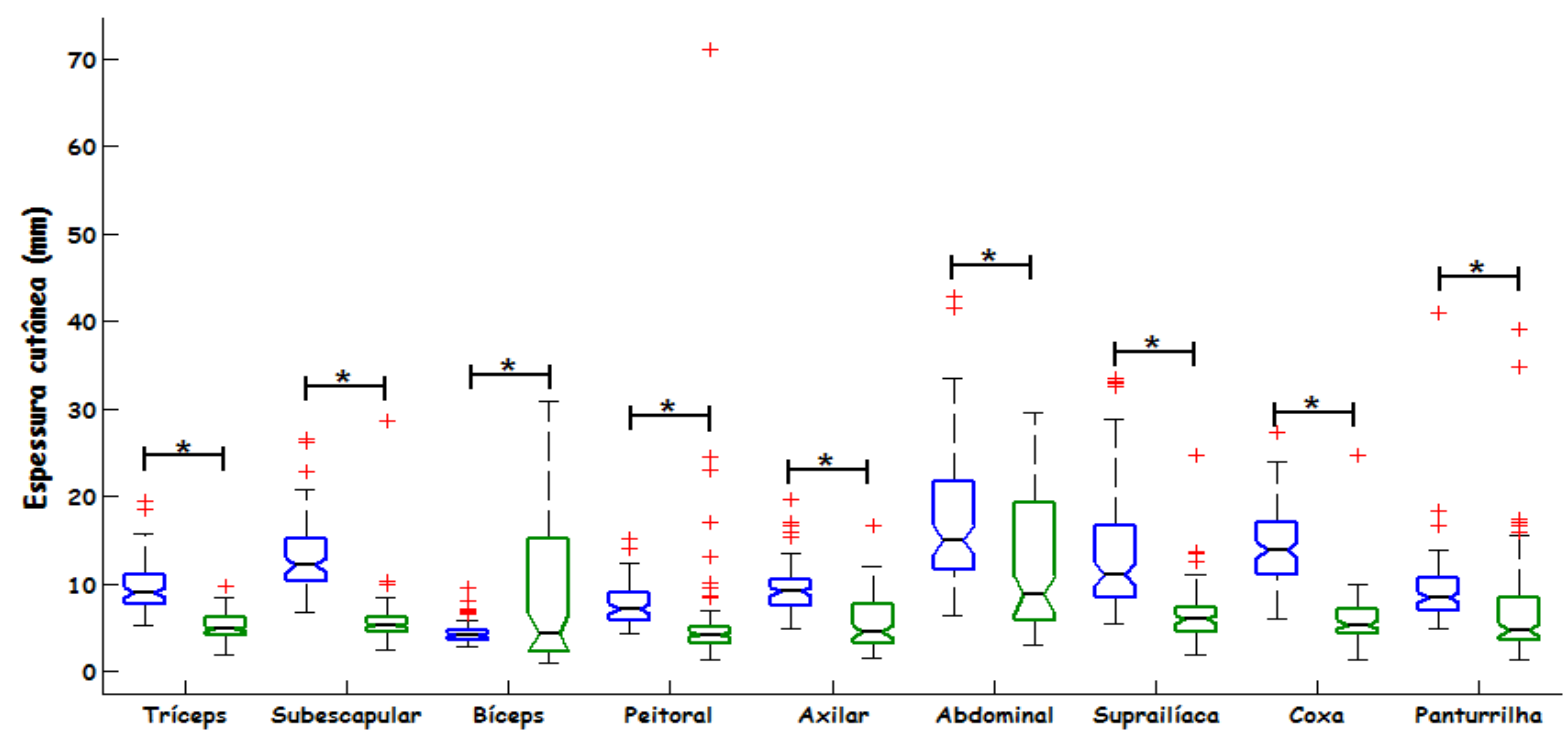

Figura 2: Gráfico do tipo boxplot da espessura cutânea registrada pelo adipômetro (cor azul) e pelo ultrassom (cor verde) em voluntários com IMC $<25 \mathrm{~kg} / \mathrm{m}^{2}(\mathrm{~N}=94)$.+: outliers. *: $\mathrm{p} \leq 0,05$.

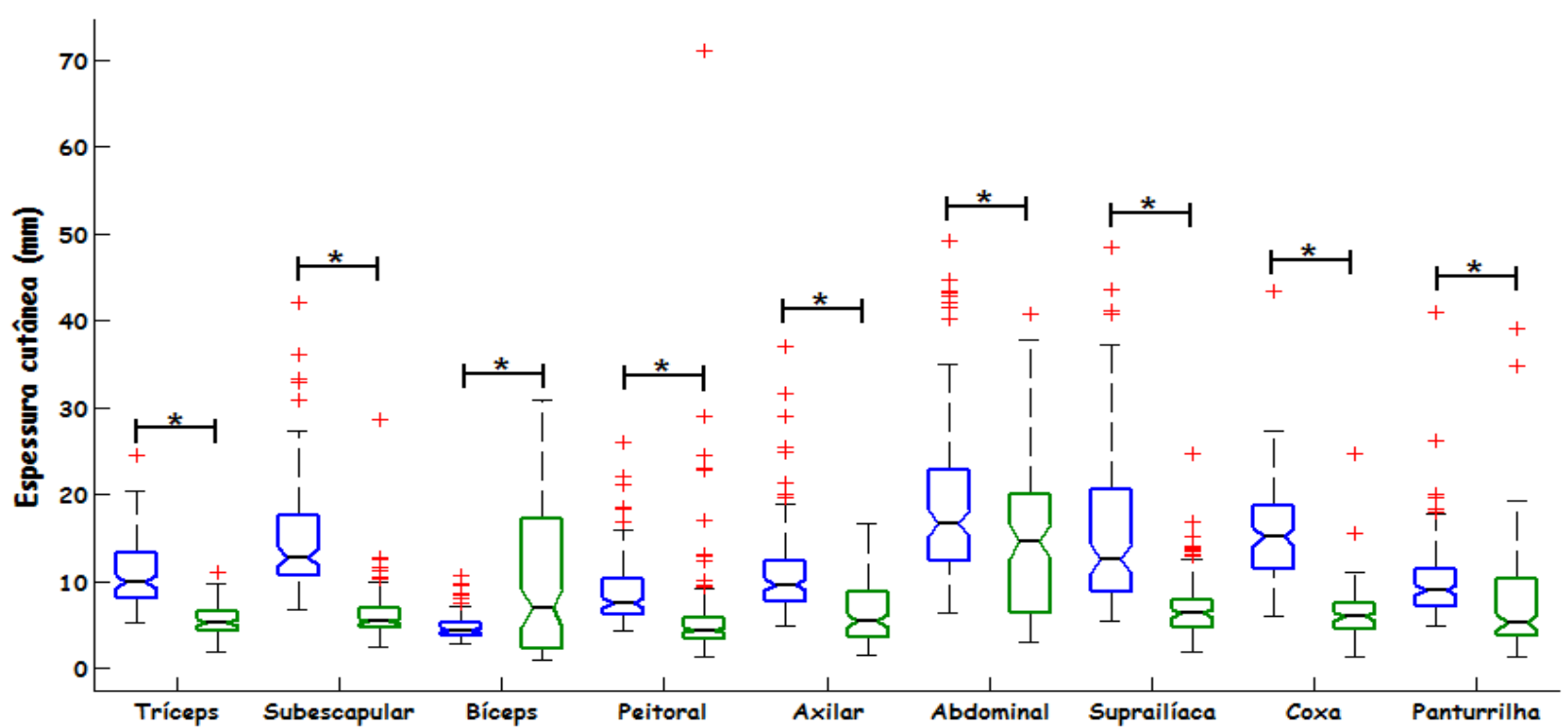

Figura 3: Gráfico do tipo boxplot da espessura cutânea registrada pelo adipômetro (cor azul) e pelo ultrassom (cor verde) em todos os voluntários $(\mathrm{N}=120)$.+: outliers. *: $\mathrm{p} \leq 0,05$.

O Wilcoxon Signed Ranks Test indicou uma diferença estatisticamente significativa $(\mathrm{p} \leq 0,05)$ entre as medidas obtidas por meio de DC e as medidas obtidas por US, conforme apresentado nas Figuras 1, 2 e 3. Os valores da mediana da dobra cutânea de bíceps, mensurado por meio do US, foram maiores em relação aos valores obtidos por meio do método de DC. Em todos os demais pontos anatômicos analisados, os valores obtidos por US foram menores.
Para a correlação dos percentuais de gordura obtidos por meio de sete dobras cutâneas e dos valores obtidos pela medição com US, foram obtidos os seguintes índices: $\rho=0,421 \quad\left(\mathrm{IMC}<25 \mathrm{~kg} / \mathrm{m}^{2}, \mathrm{~N}=94\right) ; \rho=0,419$ (IMC $\geq 25 \mathrm{~kg} / \mathrm{m}^{2}, \quad \mathrm{~N}=26$ ); $\rho=0,514 \quad$ (IMC geral, $\mathrm{N}=120)$, todas com estatística significante $(\mathrm{p}<0,05)$. A Tabela 2 apresenta as correlaçôes entre os dois métodos (US e DC), ponto a ponto anatômico. 
Tabela 2: Correlação ( $\rho$ ) entre as medidas de DC e US divididas por sítio anatômico.

\begin{tabular}{lccc}
\hline Regióes & $\begin{array}{c}\mathrm{IMC}<\mathbf{2 5} \mathbf{k g} / \\
\mathbf{m}^{\mathbf{2}} \\
(\mathbf{N}=\mathbf{9 4})\end{array}$ & $\begin{array}{c}\mathrm{IMC} \geq \\
\mathbf{2 5} \mathbf{~ k g} / \mathbf{m}^{\mathbf{2}} \\
\mathbf{( N = 2 6 )}\end{array}$ & $\begin{array}{c}\text { Geral } \\
\mathbf{( N = 1 2 0})\end{array}$ \\
Tríceps & $\mathrm{P}$ & $\rho$ & $\mathrm{P}$ \\
Subescapular & $0,57^{*}$ & $0,43^{*}$ & $0,63^{*}$ \\
Bíceps & $0,38^{*}$ & $0,42^{*}$ & $0,49^{*}$ \\
Peitoral & $0,24^{*}$ & 0,11 & $0,34^{*}$ \\
Axilar média & $0,60^{*}$ & $0,42^{*}$ & $0,62^{*}$ \\
Abdominal & $0,32^{*}$ & $-0,14$ & $0,35^{*}$ \\
Suprailíaca & $0,25^{*}$ & 0,07 & $0,28^{*}$ \\
Coxa & $0,23^{*}$ & $0,53^{*}$ & $0,39^{*}$ \\
Panturrilha & $0,55^{*}$ & $0,59^{*}$ & $0,65^{*}$ \\
\hline
\end{tabular}

${ }^{*} \mathrm{p}<0,05$

A Tabela 2 mostra que, para o grupo com IMC $\geq 25$, foram observadas correlaçôes negativas para as dobras axilar média $(\rho=-0,142)$ e para a panturrilha $(\rho=-$ $0,002)$. Quando o grupo foi analisado em sua totalidade $(\mathrm{N}=120)$, foram observadas correlaçóes significativas entre todos os pontos avaliados, sendo que as medidas que apresentaram maior e menor correlação foram as dobras cutâneas da coxa $(\rho=0,645)$ e da panturrilha $(\rho=0,232)$, respectivamente. Uma possível explicação para a baixa correlação da panturrilha pode ser a proximidade das camadas gordura-músculo e músculo-osso, que dificultam o registro pelo adipômetro. Por outro lado, no que se refere à prega da coxa, a boa correlação pode estar ligada às espessuras das camadas, uma vez que nesse local essas espessuras são relativamente menores.

\section{Discussão}

Os resultados do presente estudo apresentaram maiores correlaçóes entre o adipômetro e o ultrassom para a dobra cutânea da coxa $(\rho=0,65)$ e, por outro lado, a mais baixa correlação foi na dobra cutânea da panturrilha $(\rho=0,23)$. No entanto, mesmo com uma correlação significante, os valores registrados pelas técnicas foram distintos, como corroborado pelo Wilcoxon Signed Ranks Test. Recentemente, Loennekee et al. ${ }^{9}$ determinaram a validade do uso do US portátil como instrumento de medição do percentual de gordura, e o correlacionaram com o DEXA. Conforme este estudo, mas com avaliaçóes sobre ginastas jovens, os resultados em ambas as técnicas foram distintos $(p \leq 0,05)$, mas que se correlacionaram, com um valor de $r>0,70$.

No final da década de 1980 , Black et al. ${ }^{10}$ pesquisaram US com pulsos de alta frequência $(10 \mathrm{MHz})$, para obter estimativas de gordura subcutânea. Dez voluntários foram testados (quatro homens e seis mulheres) para a comparação da estimativa de gordura subcutânea, utilizando como padráo-ouro a tomografia computadorizada (TC) em quatro pontos localizados no abdômen. Na comparaçáo com a TC, o US subestimou os valores da espessura da camada de gordura subcutânea. Entretanto, o estudo concluiu que o sistema de medida por US pode produzir estimativas de espessura de gordura subcutânea satisfatórias, quando comparado à TC ( $r=0,76)$. Já no presente estudo, os valores de US foram inferiores aos obtidos por DC em todos os pontos analisados, exceto para o bíceps. Black et al. ${ }^{10}$ sugerem que, para pessoas obesas, o aparecimento de membrana divisória (septo) na camada de gordura poderia resultar na formação de dois ecos na aferição da medição do US. Isso reforça a opção do presente estudo pela análise estratificada da amostra em categorias do IMC.

Em um estudo recente (2009), Pineau et al. ${ }^{11}$ avaliaram 93 atletas jovens entre 18 e 33 anos (média de 23,5 anos) de ambos os sexos (vinte e quatro mulheres e sessenta e nove homens), e comparou os resultados obtidos por meio do US portátil $(5,0 \mathrm{MHz})$ e do DXA. Como resultado, esses autores verificaram que a estimativa de gordura corporal total realizada pelo US e pelo DXA obteve um alto nível de acurácia para ambos os sexos (geral: $\mathrm{r}=0,99 \pm 1,13$; mulheres: $\mathrm{N}=11 \mathrm{r}=0,98 \pm 1,61$; homens: $\mathrm{n}=35 \mathrm{r}=0,98 \pm 0,96)$. No presente estudo, o valor dos coeficientes de correlação foram inferiores $(\rho<0,70)$ aos encontrados por Pineaue et al. ${ }^{11}$. Talvez isso seja justificado pela diferença na frequência do equipamento de US utilizado, pois neste estudo a frequência do equipamento de US foi de $2,5 \mathrm{MHz}$, e como a frequência do US é inversamente proporcional à sua penetração ${ }^{12}$, e os participantes avaliados da pesquisa realizada por Pineaue et al. ${ }^{11}$ foram atletas (baixo IMC), o equipamento utilizado possuía características específicas para a aplicação nessa população.

As dobras cutâneas que apresentaram maior correlação neste estudo foram: coxa $(\rho=0,65)$; tríceps $(\rho=0,63)$; peitoral $(\rho=0,62)$ e subescapular $(\rho=0,49)$. Essa tendência foi similar ao estudo prévio conduzido por Neves et al. ${ }^{13}$ : coxa $(\rho=0,43)$; tríceps $(\rho=0,57)$; peitoral $(\rho=0,48)$; subescapular $(\rho=0,23)$, entretanto, com $\mathrm{N}=30$ e IMC entre 21 a $24 \mathrm{~kg} / \mathrm{m}^{2}$. Diferentemente desses achados, em um estudo divulgado pela empresa Intela Metrix, fabricante do equipamento, os autores Hager e Utler ${ }^{14}$ encontraram uma correlação de $\mathrm{r}=0,97$ entre a pesagem hidrostática e o US. No presente estudo, as correlações entre DC e US apresentaram valores de $\rho<0,70$ para os diversos pontos anatômicos analisados.

Em um estudo realizado por Ulbricht et al. ${ }^{15}$, tendo dois grupos divididos pela mesma metodologia do 
presente trabalho, ou seja, um grupo com IMC $<25 \mathrm{~kg} /$ $\mathrm{m}^{2}(\mathrm{~N}=30)$ e outro com IMC $>25 \mathrm{~kg} / \mathrm{m}^{2}(\mathrm{~N}=30)$, mesmo com diferenças significantes em pontos isolados de DC, comparando a aquisiçáo do adipômetro e do US com o cálculo de percentual de gordura corporal total, as duas técnicas foram similares $(\mathrm{p}>0,05)$.

Sendo assim, mesmo com diferenças isoladas entre as técnicas, de modo geral, elas descrevem resultados clínicos similares.

\section{Conclusão}

Ao compararmos os percentuais de gordura estimados pelo ultrassom (US) portátil e pelas dobras cutâneas (DC), verificamos correlaçôes significativas entre todos os pontos avaliados. Os pontos com maior correlação foram: coxa $(\rho=0,65)$ e tríceps $(\rho=0,63)$. As piores correlaçóes encontradas foram as da panturrilha $(\rho=0,23)$ e do abdominal $(\rho=0,29)$. Os valores de US foram menores do que os obtidos por DC em todos os pontos analisados, exceto para o bíceps braquial. Em vista disso, recomendamos a realização de mais estudos no futuro, comparando o US portátil e o DXA, para que se possa efetivamente verificar a existência de uma correlação entre o diagnóstico obtido a partir dessa nova tecnologia, com uma técnica considerada padrão-ouro.

\section{Agradecimentos}

Os autores agradecem à CAPES e ao CNPQ pelo suporte financeiro para a realização deste trabalho.

\section{Referências}

1. Whittingham G. Measurement of tissue thickness by ultrasound. Aerospace medicine. 1962;33:1121.

2. Wagner DR. Ultrasound as a tool to assess body fat. J obes. 2013 jul.

3. Smith-Ryan AE, Fultz SN, Melvin MN, Wingfield HL, Woessner MN. Reproducibility and Validity of A-Mode Ultrasound for body composition measurement and classification in overweight and obese men and women. PLoS ONE. 2014 jul; 9(3):e91750.
4. Booth R, Goddard B, Paton A. Measurement of fat thickness in man: a comparison of ultrasound, Harpenden calipers and electrical conductivity. Br J Nutr. 1966;20:719-25.

5. Fanelli MT, Kuczmarski RJ. Ultrasound as an approach to assessing body composition. Am J Clin Nutr. 1984;39(5):703-9.

6. Johnson KE, Miller B, Juvancic $\bowtie$ Heltzel JA, Agnor SE, Kiger DL, Kappler RM, et al. Agreement between ultrasound and dual冈energy X®ray absorptiometry in assessing percentage body fat in college冈aged adults. Clin Physiol Funct Imaging. 2014;34(6):493-6.

7. Jackson AS, Pollock ML. Generalized equations for predicting body density of men. Br J Nutr. 1978;40(3):497-504.

8. Siri WE. Body composition from fluid spaces and density: analysis of methods. Techniques for measuring body composition. Washington (DC). National Academy of Sciences. 1961:223-44.

9. Loenneke JP, Barnes JT, Wagganer JD, Pujol TJ. Validity of a portable computer囚based ultrasound system for estimating adipose tissue in female gymnasts. Clin Phys Funct Imaging 2014 set;34(5):410-2.

10. Black D, Vora J, Hayward M, Marks R. Measurement of subcutaneous fat thickness with high frequency pulsed ultrasound: comparisons with a caliper and a radiographic technique. Clin Phys Physiol Meas. 1988;9(1):57-64.

11. Pineau JC, Filliard JR, Bocquet M. Ultrasound techniques applied to body fat measurement in male and female athletes. J Athl Train. 2009;44(2):142.

12. Kitchen S. Eletroterapia: prática baseada em evidências. São Paulo: Manole; 2003.

13. Neves EB, Ulbricht L, Stadinik AMW, Matos O, Ripka W. Avaliação das medidas de composição corporal obtidas por ultra-som. In: Biomédica SBdE, ed. XXII Congresso Brasileiro de Engenharia Biomédica. Tiradentes (MG). 2010:493-6.

14. Hager M, Utler A. Bodymetrix System vs. Skinfold Caliper vs. Underwater Weighing IntelaMetrix. 2010 [cited 201019 ago 2010]; Available from: http://yardstickfitness.com/PDF/BodyMetrix \%20Validation $\% 20$ Studies.pdf

15. Ulbricht L, Neves E, Ripka W, Romaneli E. Comparison between body fat measurements obtained by portable ultrasound and caliper in young adults. Eng Med Bio Sci. 2012. Annual International Conference of the IEEE. IEEE;2012:1952-5.

\section{Como citar este artigo:}

Krueger E, Ulbricht L, Ripka W, Neves EB. Avaliação da tecnologia do ultrassom portátil e sua correlação com o percentual de gordura obtido pelas dobras cutâneas em adultos jovens. Rev. Aten. Saúde. 2015;13(46):78-83 